\title{
Locating stridor caused by tumor compression by using a multichannel electronic stethoscope: a case report
}

\author{
Fushun $\mathrm{Hsu}^{1,2}$. Cheng-Hung How ${ }^{3} \cdot$ Shang-Ran Huang ${ }^{4} \cdot$ Yi-Tsun Chen $^{4} \cdot$ Jin-Shing Chen ${ }^{5} \cdot \mathrm{Ho-Tsung} \mathrm{Hsin}^{6}$
}

Received: 19 November 2019 / Accepted: 27 April 2020 / Published online: 9 May 2020

(c) Springer Nature B.V. 2020

\begin{abstract}
A 67-year-old male patient with chronic obstructive pulmonary disease was admitted to a hospital in northern Taiwan for progressive dyspnea and productive cough with an enlarged left upper lobe tumor $\left(5.3 \times 6.8 \times 3.9 \mathrm{~cm}^{3}\right)$. Previous chest auscultation on outpatient visits had yielded diffuse wheezes. A localized stridor (fundamental frequency of $125 \mathrm{~Hz}$ ) was captured using a multichannel electronic stethoscope comprising four microelectromechanical system microphones. An energy-based localization algorithm was used to successfully locate the sound source of the stridor caused by tumor compression. The results of the algorithm were compatible with the findings obtained from computed tomography and bronchoscopy (mean radius $=9.40 \mathrm{~mm}$ and radial standard deviation $=14.97 \mathrm{~mm}$ ). We demonstrated a potential diagnostic aid for pulmonary diseases through sound-source localization technology based on respiratory monitoring. The proposed technique can facilitate detection when advanced imaging tools are not immediately available. Continuing effort on the development of more precise estimation is warranted.
\end{abstract}

Keywords Adventitious respiratory sound $\cdot$ Auscultation $\cdot$ Source localization $\cdot$ Stethoscope $\cdot$ Stridor

\section{Introduction}

Chest auscultation has had long played a useful role in physical examination well before its widespread use after the invention of stethoscope 200 years ago [1]. Respiratory

Fushun Hsu and Cheng-Hung How have equally contributed to this work.

Ho-Tsung Hsin

hsinht@gmail.com

1 Graduate Institute of Biomedical Electronics and Bioinformatics, National Taiwan University, Taipei, Taiwan

2 Department of Critical Care Medicine, Far Eastern Memorial Hospital, New Taipei, Taiwan

3 Division of Thoracic Surgery, Department of Surgery, Far Eastern Memorial Hospital, New Taipei, Taiwan

4 Department of Biomedical Engineering, National Taiwan University, Taipei, Taiwan

5 Department of Surgery, National Taiwan University Hospital, Taipei, Taiwan

6 Division of Cardiovascular Medicine, Far Eastern Memorial Hospital, No. 21, Sec. 2, Nanya South Road, 22060 Banqiao, New Taipei, Taiwan sounds are auscultated from the trachea (tracheal sounds), larger bronchial airways (bronchial sounds), or lungs (vesicular sounds). Tracheal and bronchial sounds are heard clearly during both inspiratory and expiratory phases, whereas vesicular sounds can be heard clearly only during the inspiratory and early expiratory phases [2,3]. Pulmonary pathologies are suspected when the frequency or intensity of the respiratory sounds change or when common adventitious (abnormal) sounds are identified [1,3]. Adventitious sounds can be classified into two groups according to their duration. The duration of continuous adventitious sounds (CASs) is mostly larger than $250 \mathrm{~ms}$, and the duration of discontinuous adventitious sounds (DASs) is less than $25 \mathrm{~ms}$ [3]. CASs can be further classified into subtypes such as wheeze, stridor, and rhonchus depending on their continuity, duration, phase (timing), pitch, quality, cause, and associated diseases [3]. Immediate management of abnormalities detected by timely and adequate auscultation of CASs related to airway narrowing or obstruction can prevent severe consequences or even death. Moreover, the identification of CASs and their source through auscultation enables clinicians to make clinical judgments.

Conventional auscultation has several drawbacks and limitations. First, conventional auscultation cannot be used 
continuously. [3]. Furthermore, the interpretation of the auscultation results is dependent on the practitioner's hearing ability, training, and expertise. Interpretations can vary considerably, and agreement rate among practitioners is usually fair [4-6]. Recent innovation in computerized sound analysis has increased the clinical value of auscultation by the precision improvement in sound recording and acoustic indexes. [7] Moreover, computerized analysis involving microphone arrays can be used for locating the source of adventitious sounds; however, this method has not been widely discussed. Acoustic source localization technologies have been reported extensively in the literature over the past twenty years. These technologies are categorized into the following three types according to the type of physical measurements: (1) received signal strength or energy; (2) time of arrival (TOA) or time difference of arrival (TDOA); and (3) direction of arrival (DOA) $[8,9]$. Meng and Xiao reviewed various energy-based formulations, including centralized, sequentially distributed, and fully distributed algorithms, and their corresponding problem-solving approaches to obtain single or multiple source locations [9]. Cobos et al. provided problem formulations and solutions for the TOA, TDOA and DOA models [8]. Since 2000, many studies have adopted acoustic source localization technologies to locate lung sounds. Kompis et al. proposed a method to locate lung sound and derive an acoustic image [10]. Salehin et al. used eigen-basis decomposition to locate lung sound with a circular array of sensors [11]. McKee and Goubran compared an energy-based model (identifying the location of maximum power) with a TOA model for sound localization in the human thorax [12]. Ozer et al. [13] and Henry et al. [14] have used the boundary element method to model the acoustic properties of the lung and locate the sound source in the lung. Unlike the aforementioned acoustic models, Sen, Saraclar and Kahya used an independent component analysis (ICA) and a center of weights approach to determine the location of crackles (a type of DAS) [15]. Charleston-Villalobos used ICA and a time variant autoregressive model to derive a heat map of crackle locations [16].

Adventitious lung sound localization is potentially beneficial in diagnosing pulmonary diseases because the location of the sound source can indicate the anatomical region that is most likely affected. In the present study, an energybased localization method was used to locate a persistent stridor resulting from tumor compression. A multichannel electronic stethoscope was used in the method.

\section{Materials and methods}

\subsection{The case}

A 67-year-old male chain smoker was admitted to a hospital in northern Taiwan because of an enlarged left upper lobe (LUL) lung mass and a 4-month history of shortness of breath and productive cough. A year prior to the current admission, the patient underwent thoracoscopic wedge resection and pleurodesis for left spontaneous pneumothorax. Chest auscultation on outpatient visits yielded diffuse wheezes. Treatment for the patient's chronic obstructive pulmonary disease (COPD) did not alleviate his progressive symptoms. Chest X-ray revealed no pneumothorax recurrence; however, an enlarged LUL lung mass was observed. Chest computed tomography (CT) performed 2 months prior to the current admission revealed an irregular left lingular lung mass measuring $5.3 \times 6.8 \times 3.9 \mathrm{~cm}^{3}$ with a halo sign (Fig. 1a). The patient did not experience chest pain, hemoptysis, and marked weight loss since the symptoms began. Lung malignancy was suspected. The patient was left hemiplegic due to a stroke 1 year prior to the current admission. His past medical history included hypertension, diabetes mellitus, COPD, asthma, and COPD overlap syndrome. At the time of admission, the patient was experiencing mild to moderate respiratory distress. Stridor was heard at multiple sites with a conventional stethoscope but was more pronounced over the left middle lung field. No fever was observed but the complete blood count indicated leukocytosis. The patient's chest X-ray revealed a left upper lung mass with obstructive pneumonitis. Appropriate medical treatment, including oxygen support and empirical antibiotics, was provided.

The pathology report of the patient obtained through CT-guided biopsy 1 week after admission indicated extensive necrosis, which was compatible with the ulcerative and necrotic tissues observed during bronchoscopy performed 2 days later. Tumor necrosis could not be excluded. The patient contracted right lower lobe pneumonia that soon progressed to acute respiratory failure. Adult respiratory distress syndrome subsequently developed, and the patient was intubated 2 weeks after admission. Five weeks after admission, the second bronchoscopy revealed dynamic collapse of the narrowed left inferior bronchial orifice during coughing and exertion with a widened left second carina (Fig. 1c). Suspected tumor infiltration at the left upper division caused lingular orifice occlusion (Fig. 1d); however, the cytology report indicated mainly reactive mesothelial cells and inflammatory cells. Although the patient was weaned from mechanical ventilator support and extubated 6 weeks after admission, left upper lung atelectasis persisted. The patient refused further aggressive treatment and passed away a few days later.

\subsection{Sound recording}

We recorded the chest sounds of the patient when the patient was under mechanical ventilation support. The recording device was an experimental multichannel 


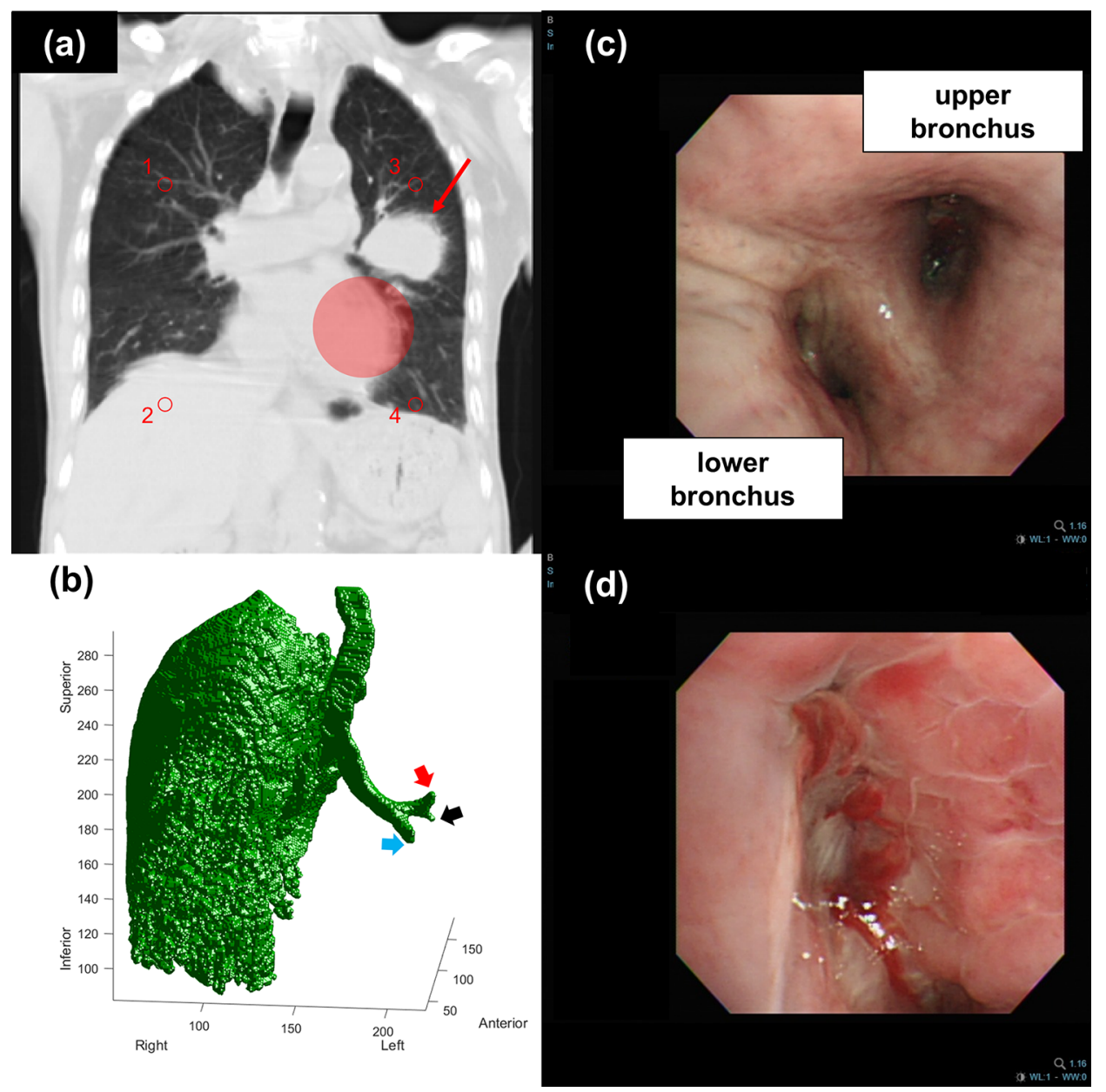

Fig. 1 CT image, airway visualization, and bronchoscopy of the patient. a Coronal view of the CT scan 2 months before admission. A tumor with dimensions of $5.3 \times 6.8 \times 3.9 \mathrm{~cm}^{3}$ at the left upper lung (red arrow). Chest sounds were recorded using four sensor patches on the second and fifth intercostal spaces along the left and right midclavicular lines (four small red circles). The source location was estimated according to 30 successive stridor signals and displayed as a visual cue (red shaded round area). The center and radius (29.94 $\mathrm{mm}$ ) of the visual cue were the mean radius value and twice the radial standard deviation of the estimated location, respectively. b The left lung is not observed in the 3D illustration of the intra-thoracic air-

stethoscope comprising four acoustic sensor patches, in each of which there was a microelectromechanical system (MEMS) microphone, SPA1687LR5H-1 (Knowles, Illinois, USA). The four sensor patches were attached on the second and fifth intercostal spaces along the left and right midclavicular lines (Fig. 1a). The sensitivity of the microphone is $-38 \mathrm{dBV} / \mathrm{Pa}$. The acoustic signal first passed through an amplifier, OPA2376AIDGKR (Texas Instruments, Texas, USA), with a gain of $18 \mathrm{~dB}$, and then an analog to digital converter, ADS8867IDGS (Texas Instruments, Texas, USA), before subsequent analysis. The sampling rate was $4,000 \mathrm{~Hz}$, and the bit depth was 16 bits. way created using a region-growing-based method. Orifices leading to the left lung (red arrow: left superior lobar bronchus; black arrow: lingular bronchus; cyan arrow: left inferior lobar bronchus) may be occluded or narrowed to ensure that the seeds are prevented from growing into the region. c Bronchoscopy performed during intensive care indicated widening of the left second carina, which implied external compression in the vicinity. d Bronchoscopy also revealed mucosa infiltration, with a cobble-stone appearance over the LUL bronchus. The lingular orifice was completely occluded by mucus and blood clots

\subsection{Signal preprocessing}

The received time signals of the four sensors were obtained as spectrograms by using the short-time Fourier transform (FT) with a Hamming window filter (window size $=512$, overlapped ratio $=0.85)($ Fig. $2 \mathrm{a}-\mathrm{d})$. An experienced physician first manually drew regions of interest (ROIs) on the spectrograms obtained from sensor 4 , which contained the stridor (white rectangles in the Fig. 2d), because the most prominent sound was recorded by sensor 4 . Then, the ROIs with the same frequency boundaries and temporal boundaries as the previously outlined ROIs were automatically 
defined on the other spectrograms (white dashed rectangles in the Fig. 2a-c). The locations of all the ROIs were confirmed through visual inspection. After determining the ROIs, we transformed the audio signals located between the temporal boundaries in each ROI into a power spectrum by using the fast FT. Figure $3(\mathrm{a}-\mathrm{d})$ displays the spectrum of the signals within the same time period bounded by one of the ROIs derived from sensor 1 to sensor 4 . The energy peak of the stridor appeared between the frequency boundaries of the ROIs in the spectrum (red lines in Fig. 3a-d). The peak value of the stridor derived from the four sensors can be used to locate the source of the sound according to an energy-ratio least-squares method described in the following section.

\subsection{Source localization}

A simple energy-based localization model with a least-square optimization [17] was used to locate each stridor. Assuming that $N$ sensors are used to receive isotropic acoustic signals propagated from a single-point sound source, the signal energy measured on the $i$ th sensor at time point $t$, which is denoted as $y_{i}(t)$, can be expressed as follows:

$y_{i}(t)=g_{i} \frac{I\left(t-\eta_{i}\right)}{\left\|\theta\left(t-\eta_{i}\right)-r_{i}\right\|^{\alpha}}+\varepsilon_{i}=s_{i}(t)+\varepsilon_{i}(t)$.

In Eq. (1), $I(\mathrm{t})$ is the energy at $1 \mathrm{~m}$ from the source; $\eta_{i}$ is the propagation delay from the source to the $i$ th sensor; $\theta(t)$ is the source location at time $t, r_{i}$ is the location of $i$ th sensor; $g_{i}$ is the gain factor of the $i$ th acoustic sensor; $\alpha$ is an energy decay factor; $s_{i}(t)$ is the energy intensity at the sensor location; and $\varepsilon_{i}(t)$ is a term that depends on the modeling errors of the parameters $g_{i}, r_{i}$, and $\alpha$ as well as the additive noise of $y_{i}(t)$. We calculated the average energy over the time window $[t-T / 2, t+T / 2]$ by using the following expression:

$y_{i}(l) \approx \frac{1}{T} \sum_{t-\frac{T}{2}}^{t+\frac{T}{2}} x_{i}^{2}(t)$, (a)

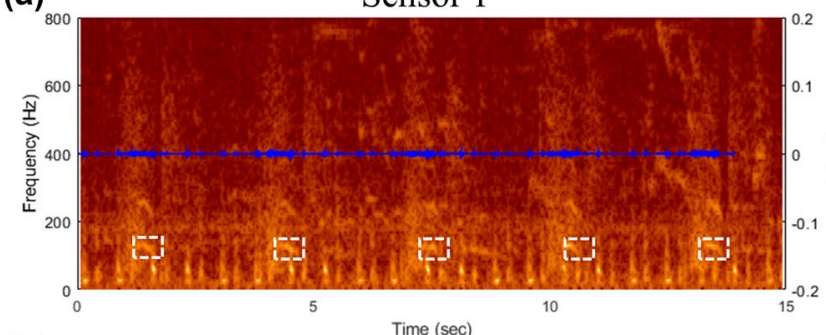

(b)

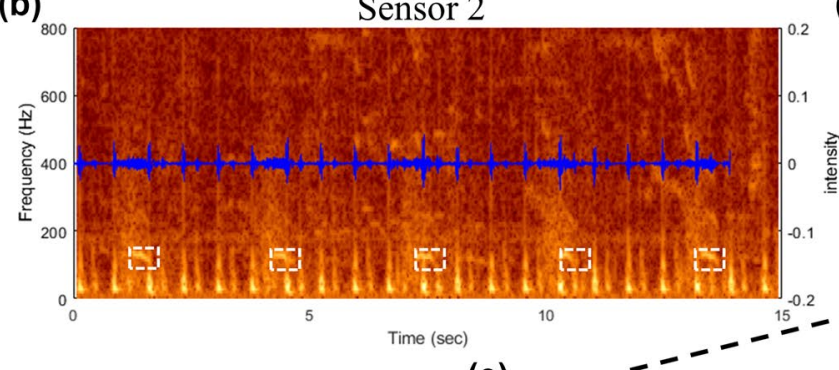

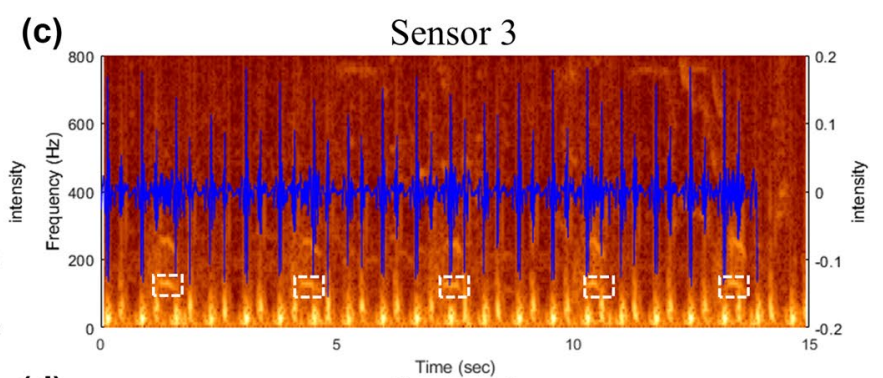

(d)

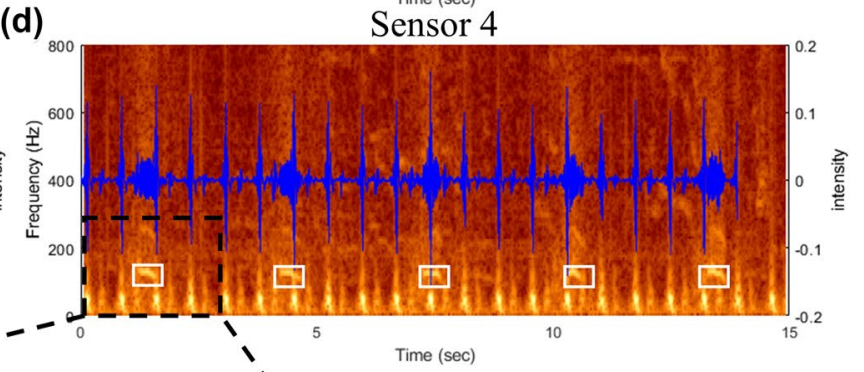

(e)

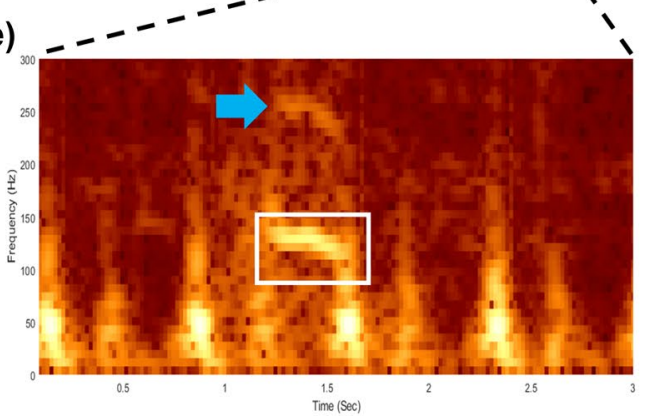

Fig. 2 Spectrograms of the collected signals from the four sensors. a-d Spectrograms of the acoustic signals collected from the four sensors. Each spectrogram depicts time-domain signals (blue waves). Stridor was initially selected manually as ROIs on the spectrograms of sensor 4, which provide a time-frequency reference (white rec- tangles). The ROIs containing stridor in the other spectrograms were defined automatically (white dashed rectangles). e Enlarged view of the black dashed rectangle obtained from sensor 4 . The cyan arrow indicates the second harmonic wave of the reference stridor 
(a)

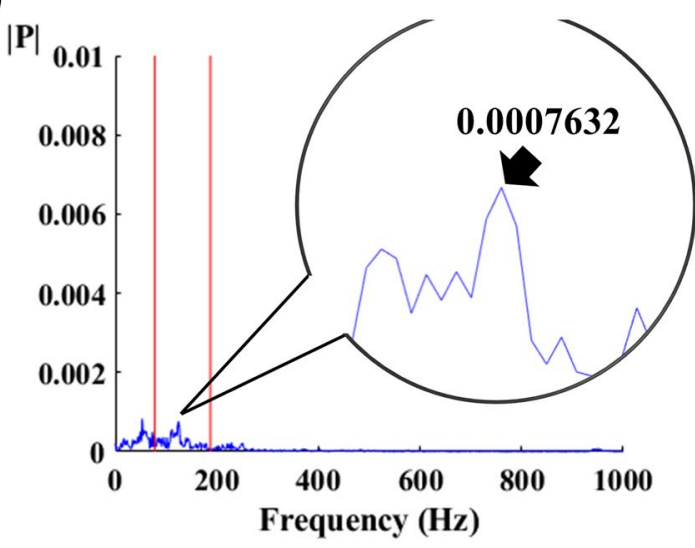

(b)

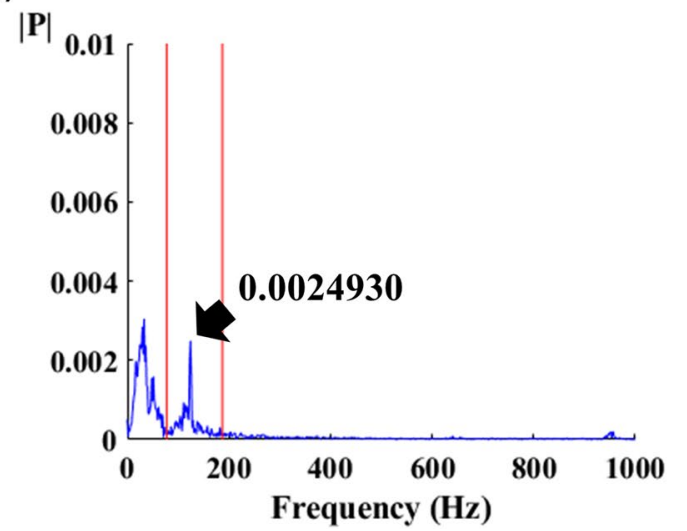

Fig. 3 Power spectrum derived according to the signals obtained from a sensor $1, \mathbf{b}$ sensor 2 , c sensor 3 , and $\mathbf{d}$ sensor 4 within the same time period bounded by one of the ROIs. The red lines denote the fre-

where $x_{i}$ is the signal intensity measured by the $i$ th sensor and $l$ is the time interval. If $T$ is comparatively large, $\eta_{i}$ can be neglected. In addition, we assumed that the source and the sensors were stationary. In this case, Eq. (2) can be expressed as follows:

$y_{i}(l) \approx g_{i} \frac{I(l)}{d_{i}^{\alpha}}+\varepsilon_{i}$.

where $d_{i}^{\alpha}$ denotes the distance between the source and the $i$ th sensor. Empirically, the decay exponent $\alpha$ is close to 2 when the sound propagation medium is air without reverberation. However, determining the precise value of the decay exponent is difficult because of the material heterogeneity in the thoracic cage and reverberation. In this report, we simply assumed that $\alpha=2$. Several approaches have been reported to solve Eq. (3) and obtain the source location. In the present study, the computing ratios of the energy peaks of the stridor were obtained using a pair of sensors. The energy ratio $k_{i j}$ of a pair of sensors can be expressed as follows: (c)

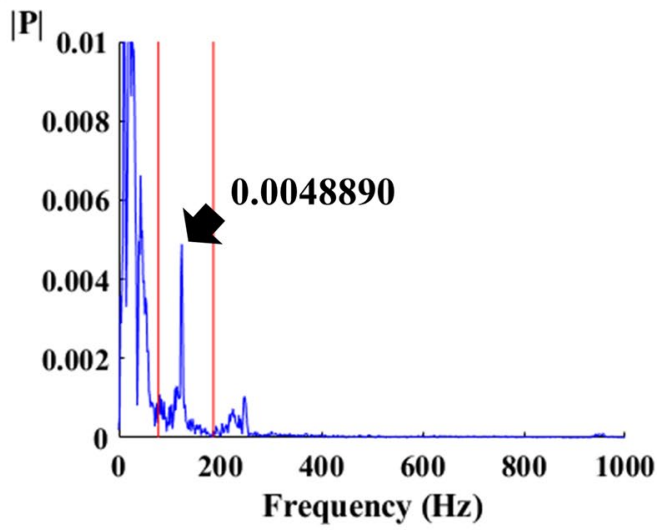

(d)

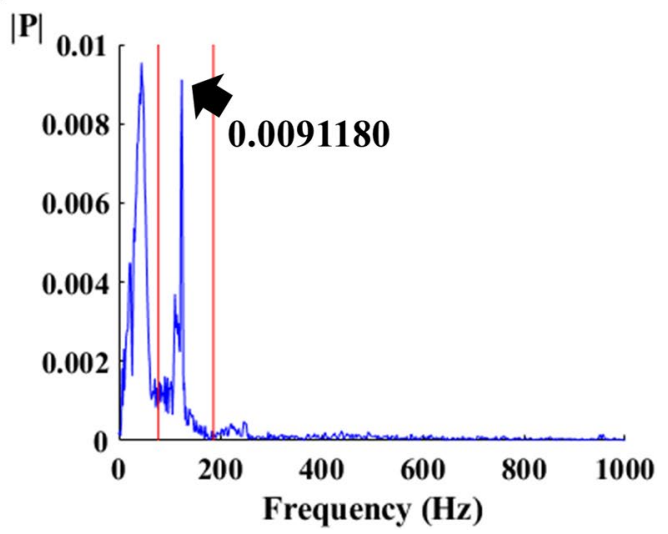

quency boundaries of the ROIs. The energy peak of the stridor should appear between the red lines in each spectrum. The values are the peak energy of the stridor (black arrows)

$k_{i j} \triangleq\left(\frac{y_{i} / y_{j}}{g_{i} / g_{j}}\right)^{-\frac{1}{2}}=\frac{\left\|\theta-r_{i}\right\|}{\left\|\theta-r_{j}\right\|}=\frac{d_{i}}{d_{j}}$

When $0<k_{i j}<1$, the coordinates of the sound source $\theta$ that satisfy Eq. (4) must reside on the circumference of a d-dimensional localizing hypersphere described by the following equation:

$\left|\theta-c_{i j}\right|^{2}=\rho_{i j}^{2}$.

The center $c_{i j}$ and radius $\rho_{i j}$ of the aforementioned hypersphere associated with sensors $i$ and $j$ are expressed as follows:

$c_{i j}=\frac{d_{i}-k_{i j}^{2} d_{j}}{1-k_{i j}^{2}}, \rho_{i j}=\frac{k_{i j}\left|d_{i}-d_{j}\right|}{1-k_{i j}^{2}}$. 
Figure 4 illustrates the six localizing hyperspheres related to four sensors and one sound source. When $k_{i j}$ $\rightarrow 1$, the solution of Eq. (4) forms a hyperplane between $d_{i}$ and $d_{j}$, which can be expressed as follows:

$\theta \cdot\left(r_{i}-r_{j}\right)=\frac{\left|r_{i}\right|^{2}-\left|r_{j}\right|^{2}}{2}$.

According to a study [17], when $\left|1-k_{i j}^{2}\right|$ is smaller than 0.001 , Eq. (7) should be used to replace equations (5) and (6) for avoiding numerical errors. When $N$ acoustic sensors are used to detect the propagated source signal during a certain time interval, $N(N-1) / 2$ pairs of energy ratios can be calculated. After acquiring $M(\leq N(N-1) / 2)$ energy ratios from these sensor pairs, we can estimate the source location $\theta$ during the time interval by solving a leastsquares optimization problem in which the objective function is defined as follows:

$J(r)=\sum_{m=1}^{M_{1}}\left|\left\|\theta-c_{m}\right\|-\rho_{m}\right|^{2}+\sum_{n=1}^{M_{2}}\left|\gamma_{n}^{T} \theta-\xi_{n}\right|^{2}$,

where $M=M_{1}+M_{2}, \gamma=\left(r_{i}-r_{j}\right)$, and $\xi=\frac{\left|r_{i}\right|^{2}-\left|r_{j}\right|^{2}}{2}$. The gradient of $J(r)$ can be expressed as follows:

$2 \sum_{m=1}^{M_{1}} \frac{\theta-c_{m}}{\left\|\theta-c_{m}\right\|}\left(\left\|\theta-c_{m}\right\|-\rho_{m}\right)+2 \sum_{n=1}^{M_{2}} \gamma_{n}\left(\gamma_{n}^{T} r-\xi_{n}\right)$.

A gradient descent method can then be used to obtain the solution of $\theta$ by minimizing the objective function in Eq. (8).

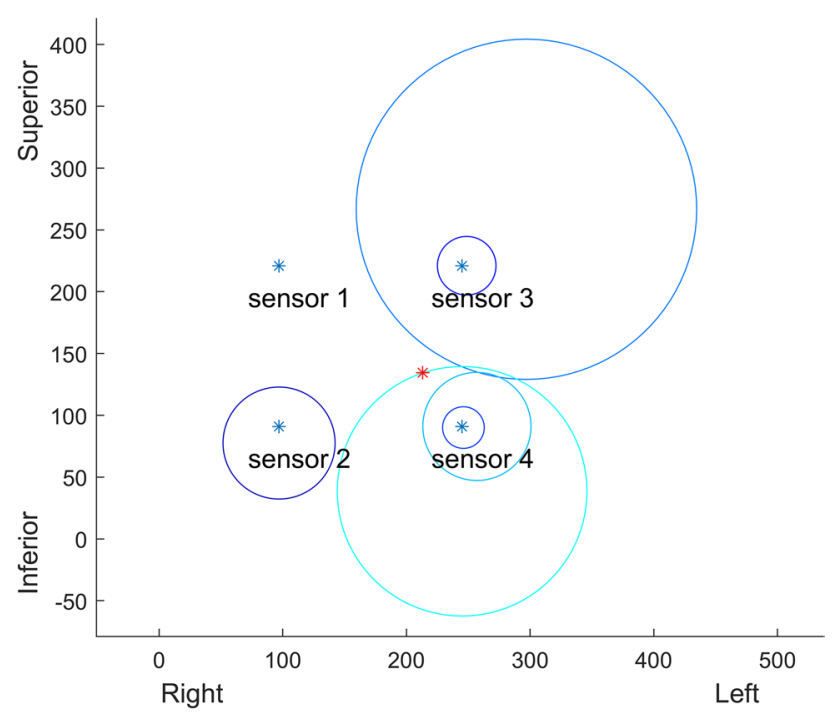

Fig. 4 Illustration of six localizing hyperspheres (colored circles) derived from four acoustic sensors (blue stars) and the estimated center of the source location (red star)
Signal processing, source localization, and visual cue display were achieved on an ASUS F302U laptop (CPU: i5-6200 U, 2.4 GHz; OS: Win10 64-bit; RAM: 8 GB; Graphics card: NVIDIA GeForce $920 \mathrm{MX}$ ) by using a platform developed through MATLAB 2019a (The MathWorks Inc., Massachusetts, USA).

\section{Results}

The source of the stridor was estimated according to 30 successive stridor signals. The mean radius and radial standard deviation were 9.40 and $14.97 \mathrm{~mm}$, respectively. A visual cue was displayed as a circular area (red shaded area in Fig. 1a) whose center and radius were the mean value and twice the radial standard deviation $(29.94 \mathrm{~mm})$ of the estimated location, respectively.

A three-dimensional (3D) illustration of the airway (Fig. 1b) was created using a region-growing-based method [18], with starting seed points placed inside the trachea according to the volumetric CT images. The absence of the left lung in the images implied nonpatency or narrowing of the airway leading to the left lung, which was compatible with the findings obtained from bronchoscopy performed during admission (Fig. 1c, d).

\section{Discussion}

Chest auscultation with a conventional stethoscope is subjective and depends mainly on the experience of the medical personnel performing the examination. In the current case, diffuse wheezes were heard on chest auscultation and was taken for granted because the patient had underlying COPD. Because of the limitation of human ears and ambient noise in the environment, identification of the focal stridor hidden underneath was difficult until image study indicated obstructive pneumonitis. Adventitious sounds can be used to study cardiopulmonary conditions. The acoustic properties of sound can be used to investigate the pathological condition of patients. Stridor is typically described as a musical sound caused by the turbulent airflow resulting from narrowing or obstruction in the upper respiratory tract, and it has a fundamental frequency of approximately $500 \mathrm{~Hz}$ [1]. In adults, stridor can indicate various pathological conditions, such as airway occlusion, narrowing, or compression, which require immediate assessment and management. Such sounds are recognizable because of the collapse of extrathoracic airways [14]. When stridor is detected in the upper respiratory tract, it can be easily distinguished from wheeze because it is more clearly heard during inhalation rather than during exhalation. However, the focal stridor derived from tumor compression of the branching bronchus in this case is 
more likely to be incorrectly identified as wheeze because of its ambiguous presentation during the expiratory phase and past medical history. By using advanced computerized sound analysis and locating technology, we observed that the sound predominantly occurred in the inspiratory phase on the spectrogram. The sound source was closer to the central airway than to the peripheral region. Therefore, a focal stridor with a relatively low frequency (fundamental frequency: $125 \mathrm{~Hz}$ ) cannot be easily missed in the proposed system.

We successfully located the sound source to be a circular area (mean radius $=9.40 \mathrm{~mm}$ and radial standard deviation $=14.97 \mathrm{~mm}$ ) by using a simple energy decay model (Fig. 1a). Since the exact location of the sound source is not obtainable, the trueness [19] of our proposed method is not reported. However, the estimated sound source covers the area including the orifice of the inferior bronchus, which is the proposed origin of the stridor. This result is in agreement with the bronchoscopy results of total occlusion in the left lingular orifice and dynamic collapse of the left inferior bronchial orifice presumably because of tumor compression. The other in vivo studies $[12,15,16]$ do not report the trueness because of the unobtainable location of sound source. On the other hand, the acoustic imaging system proposed by Kompis et al. cannot resolve the differences below about $20 \mathrm{~mm}$ on the gelatin phantom [10]. According to this standard, our proposed method performs adequately. To create a model that can more accurately locate the origin of adventitious sounds, additional acoustic parameters, such as the sound transmission path, attenuation factors of different tissue types, heterogeneity of tissues, tension of the vibration membrane of the sensor, sound leakage from the space between the skin and sound collector, and noise, should be considered. Henry and Royston proposed such a boundary element approach that models the airway tree structure, lung parenchyma and surrounding chest wall, and its accuracy can reach $2.6 \mathrm{~mm}$ to $9.8 \mathrm{~mm}$ in different simulated scenarios [14]. However, to build a boundary element model requires a CT scan to obtain the geometry of the subject's thorax beforehand.

The absence of the left lung in 3D illustration (Fig. 1b) indicated compromised airflow in the left lung. However, the actual patency of the airways leading into left lung parenchyma cannot be determined from the diagram. The partial volume effect [20] occurred because of the poor longitudinal resolution $(0.58 \times 0.58 \times 5 \mathrm{~mm})$ of the $\mathrm{CT}$ images. The voxel value of the narrowed bronchial lumen was contaminated by the Hounsfield unit of the surrounding tissues; therefore, the seeds could not grow into distal segments.

Conclusively, we demonstrated the importance and feasibility of the analysis and source localization of adventitious sounds by using a multichannel electronic stethoscope in clinic. The presented method is useful in locating an adventitious sound source at the lobular level through chest auscultation. The information regarding the sound location facilitates clinical diagnosis and treatment strategies in a noninvasive and radiation-free manner. If limited imaging modalities are available, a portable device equipped with sound-source localization software can be potentially used for diagnosis, especially when the clinical condition is progressing rapidly and critically. More effort is warranted in developing refined models in order to accommodate use in clinical practice.

Acknowledgements We thank Heroic Faith Medical Science Corporation Ltd. for providing a multichannel stethoscope. This manuscript was edited by Wallace Academic Editing.

Funding This was a self-funded research.

\section{Compliance with ethical standards}

Conflict of interest The authors declare no conflicts of interest.

Ethical approval This study was reviewed and approved by the Research Ethics Review Committee of Far Eastern Memorial Hospital (case number: 107052-F). The research was conducted in accordance with the 1964 Helsinki declaration and its later amendments or comparable ethical standards.

Informed consent Informed consent was obtained from the patient's daughter.

\section{References}

1. Bohadana A, Izbicki G, Kraman SS. Fundamentals of lung auscultation. N Engl J Med. 2014;370(8):744-51.

2. Sarkar M, Madabhavi I, Niranjan N, Dogra M. Auscultation of the respiratory system. Ann Thorac Med. 2015;10(3):158.

3. Pramono RXA, Bowyer S, Rodriguez-Villegas E. Automatic adventitious respiratory sound analysis: a systematic review. PloS ONE. 2017;12:5.

4. Behere S, Baffa JM, Penfil S, Slamon N. Real-world evaluation of the Eko electronic teleauscultation system. Pediatr Cardiol. 2019;40(1):154-60.

5. Berry MP, Martí J-D, Ntoumenopoulos G. Inter-rater agreement of auscultation, palpable fremitus, and ventilator waveform sawtooth patterns between clinicians. Respir Care. 2016;61(10):1374-83.

6. Prodhan P, Rosa RSD, Shubina M, Haver KE, Matthews BD, Buck S, Kacmarek RM, Noviski NN. Wheeze detection in the pediatric intensive care unit: comparison among physician, nurses, respiratory therapists, and a computerized respiratory sound monitor. Respir Care. 2008;53(10):1304-9.

7. Gurung A, Scrafford CG, Tielsch JM, Levine OS, Checkley W. Computerized lung sound analysis as diagnostic aid for the detection of abnormal lung sounds: a systematic review and meta-analysis. Respir Med. 2011;105(9):1396-403.

8. Cobos M, Antonacci F, Alexandridis A, Mouchtaris A, Lee B. A survey of sound source localization methods in wireless acoustic sensor networks. Wirel Commun Mob Comput. 2017;2017. https ://doi.org/10.1155/2017/3956282.

9. Meng W, Xiao W. Energy-based acoustic source localization methods: a survey. Sensors. 2017;17(2):376. 
10. Kompis M, Pasterkamp H, Wodicka GR. Acoustic imaging of the human chest. Chest. 2001;120(4):1309-21.

11. Salehin SA, Abhayapala TD. Localizing lung sounds: Eigen basis decomposition for localizing sources within a circular array of sensors. J Signal Process Syst. 2011;64(2):205-21.

12. McKee AM, Goubran RA. (2005) Sound localization in the human thorax. In: 2005 IEEE Instrumentationand Measurement Technology Conference Proceedings, 2005. IEEE, pp 117-122.

13. Ozer M, Acikgoz S, Royston T, Mansy H, Sandler R. Boundary element model for simulating sound propagation and source localization within the lungs. J Acoust Soc Am. 2007;122(1):657-71.

14. Henry B, Royston TJ. Localization of adventitious respiratory sounds. J Acoust Soc Am. 2018;143(3):1297-307.

15. Sen I, Saraclar M, Kahya YP. Acoustic mapping of the lung based on source localization of adventitious respiratory sound components. In: 2010 Annual international conference of the IEEE engineering in medicine and biology. IEEE; 2010. p 3670-3.

16. Charleston-Villalobos S, Castañeda-Villa N, Gonzalez-Camarena R, Mejia-Avila M, Aljama-Corrales T. Adventitious lung sounds imaging by ICA-TVAR scheme. In: 2013 35th annual international conference of the IEEE Engineering in Medicine and Biology Society (EMBC). IEEE; 2013. p. 1354-7.

17. Li D, Hu YH. (2003) Energy-based collaborative source localization using acoustic microsensor array. EURASIP J Adv Signal Process. 2003;4:985029.

18. Justice RK, Stokely EM, Strobel JS, Ideker RE, Smith WM. Medical image segmentation using 3D seeded region growing. In: Medical imaging 1997: image processing. International Society for Optics and Photonics; 1997. p. 900-10.

19. Menditto A, Patriarca M, Magnusson B. Understanding the meaning of accuracy, trueness and precision. Accred Qual Assur. 2007;12(1):45-7.

20. Hsieh J. Computed tomography: principles, design, artifacts, and recent advances. Bellingham; 2003.

Publisher's Note Springer Nature remains neutral with regard to jurisdictional claims in published maps and institutional affiliations. 